\title{
Designing LoRaWAN Network For Unbiased Communication Between Nodes and Gateway
}

poonam Maurya ( $\sim$ dspoonammaurya@gmail.com )

IIT Bhilai: Indian Institute of Technology Bhilai https://orcid.org/0000-0001-6357-1749

\section{Aatmjeet Singh}

IIT Bhilai: Indian Institute of Technology Bhilai

Arzad Alam Kherani

IIT Bhilai: Indian Institute of Technology Bhilai

\section{Research Article}

Keywords: LoRaWAN, Throughput, Scalability, Spreading Factor, Urbanization

Posted Date: July 19th, 2021

DOl: https://doi.org/10.21203/rs.3.rs-509474/v1

License: (c) (i) This work is licensed under a Creative Commons Attribution 4.0 International License.

Read Full License 


\title{
Designing LoRaWAN Network For Unbiased Communication Between Nodes and Gateway
}

\author{
Poonam Maurya - Aatmjeet Singh - Arzad Alam Kherani
}

Received: date / Accepted: date

\begin{abstract}
Proper cell designing is required to achieve a target system performance in Long Range Wide Area Network (LoRaWAN). This paper addresses a suitable selection of network designing parameters problem, such as the dimension of different spreading factors' annuli (zones or SF boundaries) in a LoRaWAN cell. We propose a mathematical framework for designing the LoRaWAN network. The main objective is to ensure that distributed end devices in the network can have the same success probability, irrespective of the spreading factor usage and their locations, unlike Equal Area Based (EAB) based network. We further enhance the performance of the network based on the proposed dimensions by adopting the k-tolerance algorithm. When the network follows the proposed dimensions, simulation results show an improvement in overall success probability over the traditional EAB scheme. In the later part of the paper, we address urbanization issues that degrade the system performance. In our approach to recoup the degradation in the system performance, we implement $k$-tolerance algorithm in the network.
\end{abstract}

Keywords LoRaWAN · Throughput · Scalability · Spreading Factor · Urbanization

\section{Introduction}

LoRaWAN is a widely used and well-accepted wireless communication protocol among the Low Power Wide

Poonam Maurya

E-mail: poonamm@iitbhilai.ac.in

Aatmjeet Singh

E-mail: aatmjeets@iitbhilai.ac.in

Arzad Alam Kherani

E-mail: arzad.alam@iitbhilai.ac.in
Area Network (LPWAN) protocols such as NB-IoT [7], Sigfox [23], and many others. LoRaWAN is a suitable solution for those applications that require communication over a long range with low data rate and low power requirements like smart city, agriculture, smart health center, and many more. The deployment of the LoRaWAN network is happening all over the world [5]. For example, in UK, Southampton city deployed with LoRaWAN test bed to evaluate LoRaWAN network functionality for large scale deployment [10]. The performance of LoRaWAN network highly depends on network design parameters. We can significantly enhance the network performance in terms of scalability (network capacity) and packet success probability by appropriate selection of network designing parameters like cell radius $(\mathrm{R})$ and radio resources like Spreading Factors (SF), Bandwidth (BW), Transmission Power (TP), Code Rate (CR), etc. In literature, many researchers have contributed towards optimal spreading factor allocation [15], [12], [9], [30] to improve the overall system performance of a LoRaWAN network. As the LoRaWAN network suffers from scalability and throughput issues, it is preferable to conduct a network designing based study before deploying any IoT application network.

LoRaWAN is a bi-directional MAC (Medium Access Control) layer communication protocol that adopts the Chirp Spread Spectrum (CSS) modulation technique at the physical layer (LoRa) [3]. LoRaWAN architecture is a star topology and released by the LoRa Alliance in 2015. The architecture of LoRaWAN consists of four components 1) End device, 2) Gateway, 3) Central Network Server (NS), 4) Application Server [2]. LoRaWAN operates in unlicensed spectra (Sub GHz) depending on the regional regulations. For instance, in Europe, it works around $868 \mathrm{MHz}, 915 \mathrm{MHz}$ in North America, 
920 to $928 \mathrm{MHz}$ in Japan, and 865 to $867 \mathrm{MHz}$ in India [1].

This paper estimates the LoRaWAN cell designing parameters to have unbiased (all the end devices can have the almost same success rate irrespective of SFs usage) communication with the gateway from all the end devices.

1. We proposed the LoRaWAN cell designing framework that ensures all the end devices (assigned with high and low SFs) in the network can have the same success probability. We compare the proposed dimensions with the EAB-based network dimensions where the end devices with high SF experience more collision than the end devices assigned with low SF.

2. We improve the network capacity and dimensions by implementing the k-tolerance Algorithm in the LoRaWAN network based on the proposed framework.

3. Evolution of urbanization brings some issues like high node density, congestion in signal traffic over the channel, and many more that affect the overall system performance. We put forward an approach to deal with the urbanization issues. In our approach, we are dealing with the high node density and pathloss factor by adopting $k$-tolerance algorithm.

The rest of the paper is organized as, related work is presented in Section 2. LoRaWAN main transmission parameters are presented in Section 3. The system model is described in Section 4. The description of the problem statement is given in Section 5. LoRaWAN network dimensions work has been presented in Section 6 . The system performance with tolerance Algorithm is shown in Section 7. A discussion to deal with urbanization is presented in Section 8. The numerical results of the simulation are presented in Section 9. Section 10 consists of the conclusion and discussion part of the paper.

\section{Related Work}

In [19] authors, optimized the SF boundaries, i.e., concentric ring cell around the gateway where end nodes share the same spreading factor. This optimization is based on the SNR of the end nodes available in that SF boundaries and calculated SNR corresponds to probability $H$. Whenever probability $H$ drops below targeted probability $H_{\text {target }}$ in a particular boundary, the proposed scheme modifies allocated SF for that boundary. The simulation results of the proposed scheme show significance improvement in Packet Delivery Rate (PDR) with a maximum number of nodes.
[18] develop a framework to evaluate the upper and lower bound of LoRawan network capacity, and SF boundary ranges for all SFs for IoT applications like home security. Along with optimal SF boundaries, the authors also focused on evaluating the scalability (supported number of the end devices that satisfy target bit error coverage) of LoRa network with joint noise and interference considerations.

The authors of [26] investigated network dimensions for five scenarios (Roadway signs, Traffic lights or traffic sensors, House appliances, Credit machine in a shop, and Home security) based on the considered channel attenuation model and estimated network capacity for each zone under Aloha channel assumption.

In[16] work, the authors propose two resource allocation approaches, namely 1) SensitivitySF 2) AssignmentSF to improve scalability and performance of the network. The proposed scheme adjusts each SF boundary range to have proper bidirectional communication between end devices and the gateway.

Extensive research has been done to enhance the capacity and designing parameters of the LoRaWAN network. The majority of the studies are based on optimal resource (SF) allocation. In [15], [12], [31], [11], this kind of approach has been adapted to enhance the capacity and overall system performance.

\section{LoRaWAN Protocol Transmission Parameters}

The main configurable LoRaWAN communication parameters that affect the overall network performance are described below.

\subsection{Spreading Factor}

Spreading Factor (SF) is the required number of bit used to encode a LoRa symbol. The available spreading factors for LoRaWAN communication protocols are $S F \in\{7,8,9,10,11,12\}$. In the shown Figure 1, the

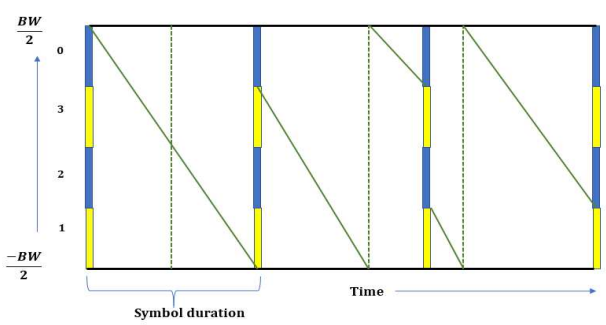

Fig. 1 Uplink Modulated LoRa Frame. 
total available bandwidth i.e. $-\frac{B W}{2}$ to $\frac{B W}{2}$ is divided into $2^{S F}$ equal number of bins. For simplicity, we take $S F=2$ in the above taken example of Figure 1. The shown bins $\{0,1,2,3\}$ corresponding to the starting frequencies for the input bit patterns $\{00,01,10,11\}$ respectively. Three symbols $\{00,11,01\}$ are being transmitted and each symbol using $S F$ number of bits (i.e., 2 bits per symbol). With the increment in SF by one step, time on air (ToA) will become double to transmit the same amount of data. One Symbol is transmitted using one complete cycle of $2^{S F}$ chips. For LoRaWAN communication, the symbol duration $\left(T_{s}\right)$ is specified as

$T_{s}=\frac{2^{S F}}{B W}$.

The LoRa packet with higher SF can travel a long distance and will have higher receiver sensitivity in comparison to the lower SF but results in low data rate as shown in Table 1. Conversely, the LoRa packet with lower SF will have high data rate, but the transmitted packet needs a higher transmission power to be properly decoded at the receiver to travel the same distance.

\begin{tabular}{|c|c|c|c|c|}
\hline SF & $\begin{array}{c}\text { chips per } \\
\text { symbol }\end{array}$ & $\begin{array}{c}\text { SNR } \\
\text { (dB) }\end{array}$ & $\begin{array}{c}\text { ToA (ms) } \\
\text { (10 byte packet) }\end{array}$ & $\begin{array}{c}\text { Bitrate } \\
\text { (bps) }\end{array}$ \\
\hline $\mathbf{7}$ & 128 & -7.5 & 56 & 5496 \\
\hline $\mathbf{8}$ & 256 & -10 & 103 & 3125 \\
\hline $\mathbf{9}$ & 512 & -12.5 & 205 & 1758 \\
\hline $\mathbf{1 0}$ & 1024 & -15 & 371 & 977 \\
\hline $\mathbf{1 1}$ & 2048 & -17.5 & 741 & 537 \\
\hline $\mathbf{1 2}$ & 4096 & -20 & 1483 & 293 \\
\hline
\end{tabular}

Table 1 SNR limit table.

\subsection{Bandwidth}

Bandwidth (BW) is one of the crucial parameter that can effect the overall sytem performance. The LoRa communication protocol can use one of the bandwidth, $B W \in\{125 \mathrm{KHz}, 250 \mathrm{KHz}, 300 \mathrm{KHz}\}$ depending on the regional parameters [1]. A LoRa modulated signal comprises of $2^{S F}$ chips, that spreads over the available bandwidth for the communication. A large value of channel bandwidth helps to achieve high data rate but with expense of more noise and interference. LoRa packet's preamble is modulated by up-chirps, whereas payload (actual data) is modulated by down-chirps for the uplink communication (end device to gateway) and vice versa for the downlink communication (gateway to end device).

\subsection{Code Rate}

Coding rate $(\mathrm{CR})$ is the degree of redundancy implemented by the forward error correction (FEC) used to detect errors in every data transmission. This implementation is done by encoding 4-bit data with redundancies into $(4+\mathrm{n})$ - bits $(n \in\{1,2,3,4\})$. Generally, Code rate for LoRaWAN protocol is defined as $\frac{4}{4+n}$. Higher value of $\mathrm{CR}$ implies that there are more error correction bits which improves protection for the transmitted data but with expense of wastage of radio resource. The parameters $S F, C R$, and $B W$ are use to calculate the bit rate $\left(R_{b}\right)$ for LoRa modulation

$R_{b}=S F * \frac{B W}{2^{S F}} * C R$.

\subsection{Transmission Power}

One of the governing parameter for LoRaWAN communication is Transmission Power (TP). LoRa network can operate with transmission power from $2 \mathrm{dBm}$ to $20 \mathrm{dBm}$ [22]. Transmission power used by an end-device has an effect on the other end-devices' transmission in terms of interference.

\subsection{Power Consumption Aspect of LoRaWAN}

For IoT communication protocol, LoRaWAN is considered a prime choice due to its long-life service. LoRa uses Spread Spectrum modulation to support low power characteristics to provide long-range communication [21]. LoRaWAN Class A devices can operate up to 10 years [6] [29]. For instance, if a LoRa device with a battery capacity $133,200 \mathrm{~J}(10,000 \mathrm{mAh})$ can transmit 288 packets per day with the expected device life time of 9.9 years [29]. The range of sleep current for LoRawAN devices is $7.66 \mu \mathrm{A}$ to $34 \mathrm{~mA}$ [13]. When we plan to setup an IoT network, it is preferable to consider the fact of power consumption. Total current consumption for a LoRa end device over a duty cycle can be defined as

$I_{\text {total }}=\frac{L_{z} I_{T x}+\frac{1}{\lambda_{0}} I_{s}}{L_{z}+\frac{1}{\lambda_{0}}}$,

where, $I_{T x}$ and $I_{s}$ are transmitting and sleeping mode current respectively. $\lambda_{0}$ is the average packet generation rate and $L_{z}$ is the transmitting time or ToA. From [13], the transmitting and the sleeping current for the LoRaWAN Multitech mDot device are $36 \mathrm{~mA}$ and 30.09 , $\mu \mathrm{A}$ respectively. At $\lambda_{0}=1$ packet per day and packet size of 250 byte with 0.4005 second ToA, an end device consumes $I_{\text {total }}=31.06 \mu \mathrm{A}$ for $S F=7$. The packet generation rate is the same for all the devices (as all devices 
are dedicated to the same application). With increment of one step in SF, the transmitting current consumption will be doubled for an end device as $L_{z+1}=2 L_{z}$. If IoT applications have low packet generation rate $\lambda_{0}$ in the system, the overall average current consumption does not vary significantly across the devices using different SFs. Hence we can conclude that as long as $\lambda_{0}$ is low, transmitting current (power) consumption will not matter.

\section{System Model}

We consider LoRaWAN single cell where zones' boundaries $\left\{d_{7},\left(d_{8}-d_{7}\right),\left(d_{9}-d_{8}\right),\left(d_{10}-d_{9}\right),\left(d_{11}-d_{10}\right),\left(d_{12}-\right.\right.$ $\left.\left.d_{11}\right)\right\}$ are in the form of concentric rings around a centrally deployed gateway as shown in Figure 2. Considered cell is divided into multiple zones $z \in\{7,8,9,10,11,12$ In a particular zone $z$, all the end devices are allocated with the same $S F \in\{7,8,9,10,11,12\}$. In our system model, end devices are uniformly distributed with spatial node density $\rho$ (nodes per kilometer square). For a given node density, all the end devices in a particular zone $z$ have the same packet duration $L_{z}$ (Time on Air). While farther end devices (lie in zone $z+1$ ) have larger packet duration for the same packet size as farther end devices use higher $S F$. The transmission of the packet from each end device is independent of each other. The total packet transmission rate in a zone $z$ is $\lambda_{z}=\lambda_{0} K_{z}$ where, $K_{z}=\rho A_{z}$ is the total number of end devices in a zone $z$ and $A_{z}=\pi\left(d_{z}^{2}-d_{z-1}^{2}\right)$ is the area of the zone $z$. In this paper, we assume that end

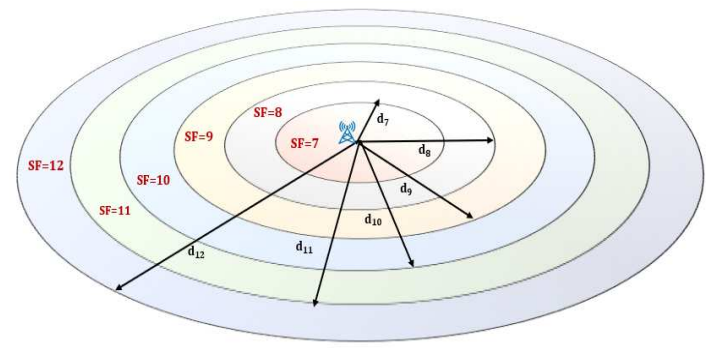

Fig. 2 A single LoRaWAN cell.

devices access the available channel in a Slotted Aloha manner, i.e., transmissions from end devices are allowed only at the beginning of the transmission slot frame of length $L_{z}$, as shown in Figure 3. We assume each end device in the network transmits with the same power $P_{0}$. The transmission slot length for a device depends on SF usage. For instance, an end device with $S F=8$ will have a double slot frame length as compared to an end device with $S F=7$. All the end-devices use the
Table 2 Table of Notation

\begin{aligned} \hline$z &$ Zone in the cell \\ $L_{z} &$ Time on air of an end device's packet lies in zone z \\ $d_{z} &$ distance of the boundary of zone z from the gateway \\ $K_{z} &$ Total number of nodes in the zone $z \\ A_{z} &$ Area of the zone $z \\ \rho &$ spatial node density \\ $\lambda_{0} &$ Packet generation rate of individual end device \end{aligned}

same channel of $B W=125 \mathrm{KHz}$ to communicate with the gateway and have perfect orthogonality, i.e., a centrally deployed gateway can receive transmissions from two different nodes with different SF's at the same time. It is observed in [24] that intra-SF interference (interference between same SF) is more dominant than inter-SF interference (collision between different SF). The signals with intra-SF interference effect have more SINR \}threshold value to demodulate than inter-SF interference. Inter-SF interference happens due to the quasi orthogonal nature of SFs [14]. In the Slotted aloha sys-

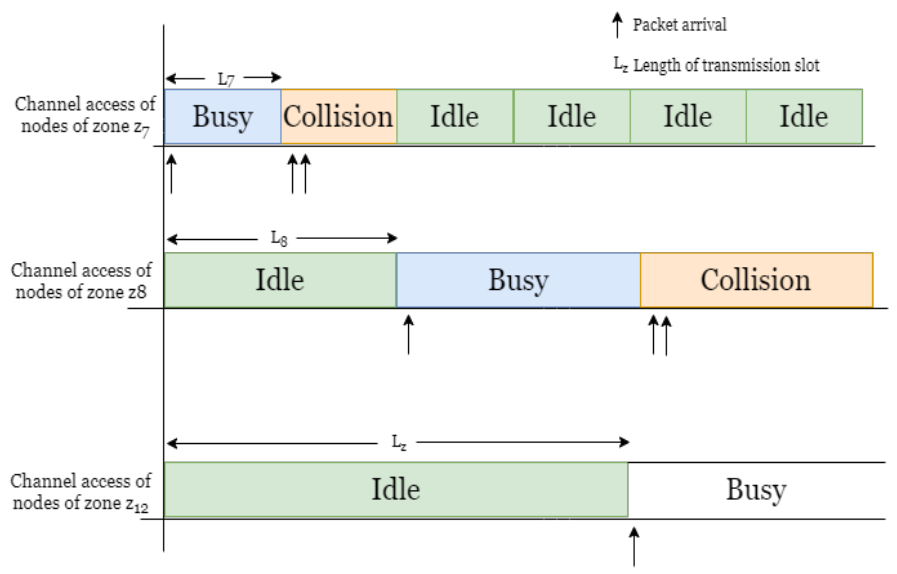

Fig. 3 Slotted Aloha Channel Access for each SFs.

tem, a collision may occur when more than one end devices start the transmission in the same transmission slot, as shown in Figure 3.

\section{Problem Statement}

In this paper, we address two LoRaWAN network challenges. First, in EAB (Equal Area Based) SF allocation [24] where LoRaWAN single cell has the same $\left(A_{7}=A_{8}=A_{9}=A_{10}=A_{11}=A_{12}\right)$ SFs zones' area. Zones have the same number of end devices if the distribution of end devices is uniform. End devices that are far away from the gateway are allocated higher SF, and the end devices nearer to the gateway are assigned lower SF. The farther end devices (assigned with higher 
SF) experience more collision probability as they have higher $L_{z}$ than the other end devices (assigned with low $\mathrm{SF})$. In this work, we estimate the network designing parameters (relation between $\left\{d_{7}, d_{8}, d_{9}, d_{10}, d_{11}, d_{12}\right\}$ ) such that each end device in the considered system model experiences the same collision probability. Using our proposed model, we ensure that the overall throughput of the individual end devices becomes independent of the SFs usage.

The Second challenge is that urbanization in the LoRaWAN network leads to change in the network characteristics like channel propagation, more deployment of IoT applications, etc. As these factors change, the network requires additional infrastructure installation (more deployment of the gateway) to avoid compromise in performance level. To deal with this, we propose an approach so that network can sustain the same performance over the time.

\section{LoRaWAN Network Dimensions}

In the considered Slotted Aloha system model, packet transmission from each end device follows the Poisson Distribution (PD). A transmitted packet in any particular slot will be successful only when there is no other transmission going on in that slot with the same SF. The success probability $P_{z}^{\text {success }}$ of the transmitted packet from an end device $i$ that lies in the zone $z$ (has total $K_{z}+1$ end devices) can be defined as

$$
P_{z}^{\text {success }}(i)=e^{-\lambda_{0} K_{z} L_{z}} \quad 1 \leq i \leq K_{z}
$$

Now we estimate LoRaWAN network designing parameters such that all the end devices in zone $z \in$ $\{7,8,9,10,11,12\}$ can have the same success probability irrespective of $z$ or $S F$. We know the relation between slot length of both end devices that lie in zone $z$ and $z^{*}=z+1$, i.e., $L_{z^{*}}=2 L_{z}$ where, $z \in\{7,8,9,10,11\}$. Equating the success probabilities of two end devices $i$ and $j$ lie in the zone $z$, and zone $z^{*}$ respectively to get the required network designing parameters

$$
\begin{aligned}
& P_{z}^{\text {success }}(i)=P_{z^{*}}^{\text {success }}(j) \\
& K_{z} L_{z}=K_{z^{*}} L_{z^{*}}
\end{aligned}
$$

We have evaluated the above equation for $z \in\{7,8,9,10,11$ and obtained the required dimensions. $d_{8}=\sqrt{\frac{3}{2}} d_{7}$, $d_{9}=\sqrt{\frac{7}{4}} d_{7}$ and $d_{10}=\sqrt{\frac{15}{8}} d_{7}$ and $d_{11}=\sqrt{\frac{31}{16}} d_{7}$ and $d_{12}=\sqrt{\frac{63}{32}} d_{7}$.

With the estimated network dimensions (i.e., $\left.d_{7}, d_{8}, d_{9}, d_{10}, d_{11}, d_{12}\right)$ we can have new relation between $\left\{A_{7}, A_{8}, A_{9}, A_{10}, A_{11}, A_{12}\right\}$ such that all the end devices have same success and collision probability. $A_{8}=\frac{1}{2} A_{7}$ and $A_{9}=\frac{1}{4} A_{7}$ and $A_{10}=\frac{1}{8} A_{7}$ and $A_{11}=\frac{1}{16} A_{7}$ and $A_{12}=\frac{1}{32} A_{7}$

$A_{7}: A_{8}: A_{9}: A_{10}: A_{11}: A_{12}=32: 16: 8: 4: 2: 1$

If we follow this kind of structural relations between different zones while deploying any LoRaWAN network, we can have unbiased communication from all end devices, unlike EAB LoRaWAN network. The estimated network designing parameters indicate that as we move from lower to higher SF, network SFs' zones area decreases, i.e., fewer devices allocated higher SF. The above derived structural results allow more end devices to use low SF to improve overall system collision probability. The aggregated throughput of zones increases as we move from the higher zone ( end devices with high SF) to the lower zone (end devices with low SF). The proposed framework allows more end devices to use low SF, but the individual end devices throughput will be the same. In the considered LoRaWAN cell model, $d_{z}^{\max }$ indicates the distance of an end device that is located at the edge of the SF boundary from the gateway. The maximum coverage zones boundaries are estimated while considering the channel attenuation model same as [18]

$\Gamma_{z}=P_{0}+G_{A N T}-K_{\text {intercept }}-10 \eta \log \left(d_{z^{\max }}\right)-P_{\text {noise }}$,

where $\Gamma_{z}$ is the average received SNR for successful reception for an end device using $S F=z$ and located at the zone $z$ edge, $G_{A N T}$ is the antenna gain of the LoRa gateway and is set to $2 \mathrm{~dB}, K_{\text {intercept }}=128.95$ is the intercept of pathloss model [27], $\eta$ is the path-loss exponent factor, and $P_{\text {noise }}=174-10 \log (B W)-N F$ $\mathrm{dBm}$ is the background noise and Noise Figure $N F=6$ $\mathrm{dBm}$. All end devices transmit with the same power $P_{0}=14 \mathrm{dBm}$.

\section{Network dimensions with k-tolerance algorithm}

In our previous work, we have proposed a $k$-tolerance Algorithm or k-multilevel Algorithm [20]. This algorithm allows multiple end devices to communicate at the same time over the same channel, i.e., a receiver implementing this algorithm can detect a known target signal in the presence of other signals. In the literature, there are some other algorithms [28], [17], [8] which achieve the same objective, i.e., allow a target number of concurrent transmissions over the same channel in the LoRaWAN network. The complexity of implementing such an algorithm increases wrt. $k$. By considering this kind of tolerance algorithm in our proposed scheme, we can further 
enhance the system performance in terms of network capacity and throughput.

\section{1 k-tolerance Algorithm}

A receiver equipped with a $k$-tolerance algorithm can receive and decode a signal of interest in the presence of other $k+1$ interfering signals over the same channel without collision. For instance, for $k=1$, a receiver (gateway) can decode a target signal in the presence of another signal over the same channel. We consider the same slotted aloha system model as described in section 4. Estimate LoRaWAN cell designing parameters to ensure that all end devices in the network with low and high value of SFs can communicate with the gateway with the same success rate.

A transmission from an end device $i$ deployed in zone $z$ of the network where a central gateway equipped with the $k$-tolerance algorithm can have collision in the transmitting slot if more than $k+1$ other devices also start the transmission in the same slot. Then success probability of a transmitted packet from an end device $i$ is defined as

${ }_{k} P_{z}^{\text {success }}(i)=\sum_{i=0}^{k} \frac{\left(\lambda_{0} K_{z} L_{z}\right)^{i} e^{-\lambda_{0} K_{z} L_{z}}}{i !}$

To estimate the required network designing parameters, we need to equalize the different zone's success probability i.e., ${ }_{k} P_{z}^{s u c c e s s}(i)$ and ${ }_{k} P_{z^{*}}^{s u c c e s s}(j)$.

${ }_{k} P_{z}^{\text {success }}(i)={ }_{k} P_{\left(z^{*}\right)}^{\text {success }}(j)$

A solution for the above equation (2) is $K_{z} L_{z}=K_{z^{*}} L_{z^{*}}=$ $c$, where $c$ is constant. This is the common solution for equations (1) and (2). Now we can conclude that system with and without a $k$-tolerance algorithm can have the same network dimensions, but network with a $k$ tolerance will have more system throughput as $k+1$ number of end devices allow to transmit in the same transmission slot unlike the network without $k$-tolerance. We consider three scenarios for LoRaWAN single cell, 1) Small cell radius 2) Medium cell radius 3) Large cell radius.

- Scenario 1 In small cell radius, all the end devices require to meet 50 percent of the average $\operatorname{SNR~} \Gamma_{z}$ threshold to have proper communication with the central gateway.

- Scenario 2 For medium cell radius, end devices should meet 75 percent of the average SNR $\Gamma_{z}$ level to have proper demodulation at the gateway.

- Scenario 3 Similarly, for a large cell radius, the end devices need to meet 100 percent of the average SNR $\Gamma_{z}$ as defined in [18] for appropriate communication.
Each scenario has the same individual packet generation rate $\lambda_{0}=1$ packet per day with packet size of 17 byte and node density $\rho=100$ nodes per kilometers square. We have considered pathloss factor $\eta=2.4$ to estimated network dimensions for small, medium, and large cell as shown in Table 3, 4, 5 respectively. Accord-

\begin{tabular}{|c|c|c|c|}
\hline $\begin{array}{c}\text { Spreading } \\
\text { Factor }\end{array}$ & $\begin{array}{c}\text { Zones dimension } \\
\left(d_{z^{\max }}\right) \mathbf{( k m )}\end{array}$ & $\begin{array}{c}d_{z_{(k=0)}} \\
\mathbf{( k m})\end{array}$ & $\begin{array}{c}d_{z_{(k=1)}} \\
(\mathbf{k m})\end{array}$ \\
\hline $\mathbf{7}$ & 0.7931 & 0.7931 & 0.79314 \\
\hline $\mathbf{8}$ & 1.2964 & 0.9713 & 1.11267 \\
\hline $\mathbf{9}$ & 1.6507 & 1.0485 & 1.57345 \\
\hline $\mathbf{1 0}$ & 1.8967 & 1.0858 & 2.22512 \\
\hline $\mathbf{1 1}$ & 2.1894 & 1.1036 & 3.1467 \\
\hline $\mathbf{1 2}$ & 2.5231 & 1.1128 & 4.45011 \\
\hline
\end{tabular}

Table 3 Network Dimensions for scenario 1.

\begin{tabular}{|c|c|c|c|}
\hline $\begin{array}{c}\text { Spreading } \\
\text { Factor }\end{array}$ & $\begin{array}{c}\text { Zones dimension } \\
\left(d_{\boldsymbol{z}^{\max }}\right) \mathbf{( \mathbf { k m } )}\end{array}$ & $\begin{array}{c}d_{\boldsymbol{z}_{(k=0)}} \\
\mathbf{( \mathbf { k m } )}\end{array}$ & $\begin{array}{c}d_{\boldsymbol{z}_{(k=1)}} \\
\mathbf{( \mathbf { k m } )}\end{array}$ \\
\hline $\mathbf{7}$ & .86811 & 0.86811 & 0.86811 \\
\hline $\mathbf{8}$ & 1.3329 & .9774 & 1.122769 \\
\hline $\mathbf{9}$ & 1.7346 & 1.1476 & 1.5872 \\
\hline $\mathbf{1 0}$ & 2.1366 & 1.1885 & 2.2446 \\
\hline $\mathbf{1 1}$ & 2.6416 & 1.2079 & 3.1734 \\
\hline $\mathbf{1 2}$ & 3.2781 & 1.2180 & 4.5387 \\
\hline
\end{tabular}

Table 4 Network Dimensions for scenario 2.

\begin{tabular}{|c|c|c|c|}
\hline $\begin{array}{c}\text { Spreading } \\
\text { Factor }\end{array}$ & $\begin{array}{c}\text { Zones dimension } \\
\left(d_{\boldsymbol{z}_{\text {max }}}\right) \mathbf{( \mathbf { k m } )}\end{array}$ & $\begin{array}{c}d_{z_{(k=0)}} \\
(\mathbf{k m})\end{array}$ & $\begin{array}{c}d_{z_{(k=1)}} \\
(\mathbf{k m})\end{array}$ \\
\hline $\mathbf{7}$ & 1.040498 & 1.0404 & 1.0404 \\
\hline $\mathbf{8}$ & 1.37792 & 1.274 & 1.4713 \\
\hline $\mathbf{9}$ & 1.822955 & 1.375 & 2.0808 \\
\hline $\mathbf{1 0}$ & 2.406941 & 1.424 & 2.9427 \\
\hline $\mathbf{1 1}$ & 3.193819 & 1.448 & 4.1616 \\
\hline $\mathbf{1 2}$ & 4.259027 & 1.459 & 5.8853 \\
\hline
\end{tabular}

Table 5 Network Dimensions for scenario 3.

ing to the LoRaWAN Specification documentation (released by LoRa Alliance) [4], the LoRaWAN communication protocol provides long-range communication: up to 5 kilometers for the urban area and up to 10-15 kilometers for the rural area (line of sight). The appropriate selection of transmission parameters helps in achieving the required range of communication in LoRaWAN. In Table 3,4 , and $5, d_{z}^{\max }$ indicates the maximum outer boundary radius for zone $\mathrm{z}$ to have a Bit Error Rate (BER) performance of $10^{-5}$ [18]. The designing parameters $d_{z_{(k=0)}}$, and $d_{z_{(k=1)}}$ are the outer boundaries radius for the zone $z \in\{7,8,9,10,11,12\}$ when network follows our proposed structural result with $k=0$ and 
$k=1$ respectively. From the estimated structural result, we observe that it is possible to increase further the cell radius ( wrt. $d_{z^{m a z}}$ ) when the network adopts the proposed designing parameters with $k=1$.

\section{Approach to deal with urbanization issues}

LoRaWAN end-devices are expected to be working for twenty years. They are of low cost and inexpensive that the idea of software-upgrading them is not feasible. Thus, once deployed, the network with the legacy devices has to function appropriately for $20+$ years. However, it is expected that the traffic generation pattern of a device may not change significantly over time as it is tied to a particular application. The many regionspecific duty cycle requirements [1] would ensure that the individual devices, once deployed, behave in the same manner throughout their 20 years lifespan. LoRaWAN system design requires careful radio planning. Being a single frequency network, the distance between the gateways is required to be as large as possible within the standard coverage constraints. However, with rapid urbanization as shown in Figure 4, several input variables to the design problem change over time, including a) the channel propagation (path loss) characteristics, and b) the device density. With these two factors changing over time, the network designed to work for now will not be able to sustain the same performance level, thus creating coverage holes in the network. One way to fix this problem is to install more gateways to cover these spatial regions which are no longer able to communicate with the existing infrastructure.

Using the signal processing techniques developed by us [20], we argue that additional infrastructure installation can be avoided in order to keep up with the rapid urbanization of the LoRaWAN-enabled area. One only has to (software/hardware) upgrade some of the gateways and perform the right spreading factor allocation. The approach is to dimension the network (appropriate dimensions of different zones, gateway placements and parameter allocation) and then keep on upgrading the receiver (gateway) capability via a signal processing up-gradation by using the k-tolerance mechanism.

8.1 Adaptation of $\mathrm{k}$ with the variation of node density $\rho$ to achieve the target system performance

Urbanization leads to more IoT application deployment. Consequently, the number of end devices increase drastically that burdens the channel traffic. The target system performance $\left(T_{p}\right)$ ) decreases with an increase in
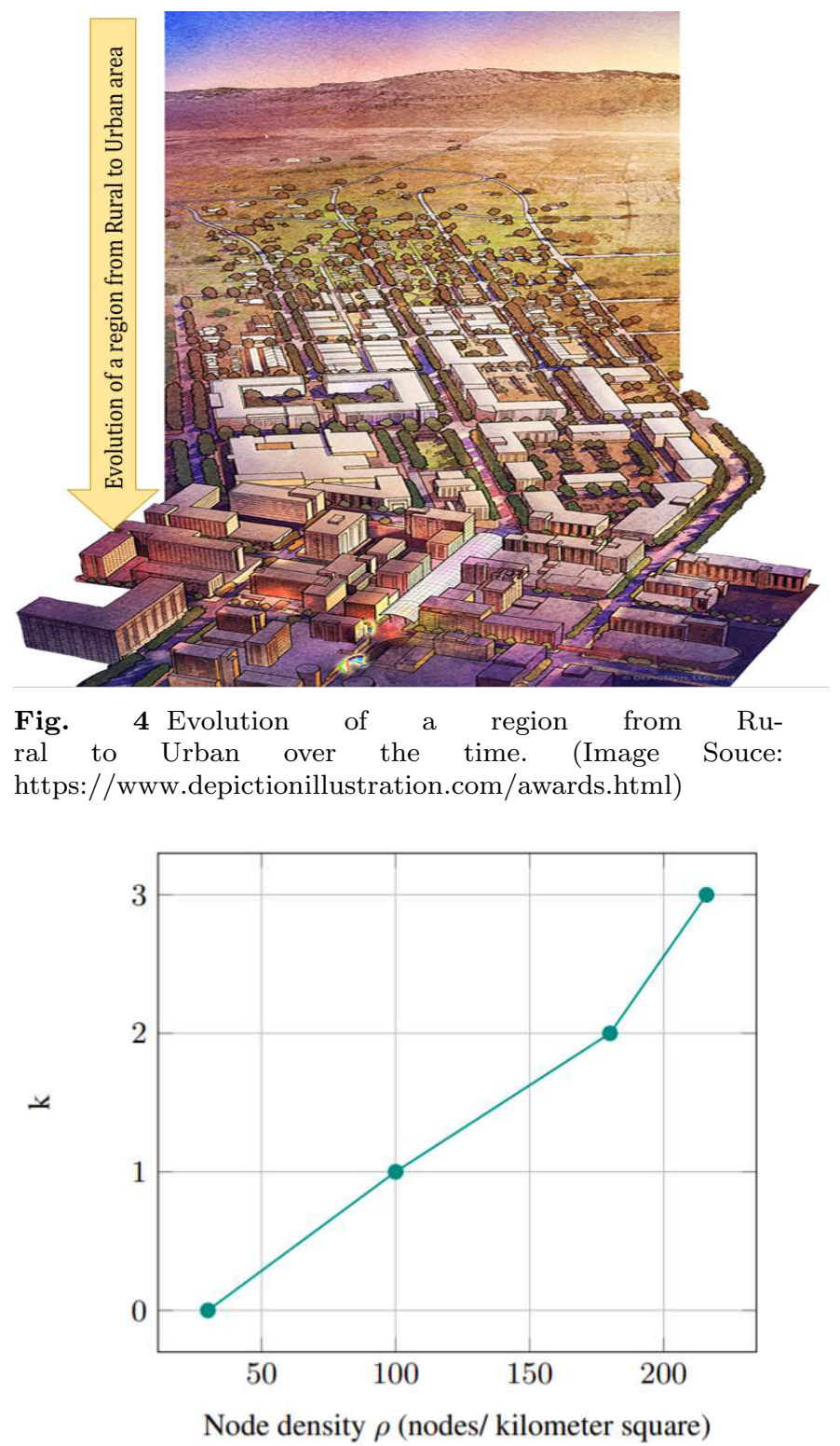

Fig. 5 Required minimum $k$ to achieve a target success probability for a given $\rho$.

IoT application deployment $(\rho)$ that leads to more collisions in the network.

$\frac{d T_{p}}{d \rho}<\left.0\right|_{k=0}$.

A possible approach to sustain the same target performance level is by implementing the $k$-tolerance algorithm [20] at the gateway, as described in Section 7.

Figure 5 depicts how the network can adapt $k$ tolerance algorithm to deal with high node density. It shows minimum value of $k$ required to maintain same performance level for a given node density. For instance, if node density increases from 30 to 100 nodes per kilometer square, we can still achieve the same target success probability of 0.95 by adopting a $k$-tolerance algorithm 
$(k=1)$. For the above example, the minimum required $k=1$, but we can also use $k>1$ to achieve the same and even higher target success probability but with the expense of more signal processing at the gateway. The complexity of implementing $k$-tolerance algorithm increases wrt. $k$ as more $k$ allows the network for more concurrent transmissions.

8.2 Adaptation of $k$ as pathloss factor varies to maintain the target system performance

One of the consequences of urbanization is an increase in pathloss factor. In this section, we present a theorem which validates that a network equipped with the $k$-tolerance algorithm can cope with the pathloss factor $\eta$ variation to achieve same performance level.

In Figure 6, end devices access the channel in a slotted Aloha manner (transmissions can be initiated only at the beginning of the transmitting slot, i.e., slot-1, slot-2, slot-3, slot-4, and slot-5). Each transmitting slot has one target packet and some interfering packets. Network trace in Figure 6 indicates network's traffic in slot 1 to 5 . It shows the behavior of the target packet (success or collision) when LoRaWAN network is equipped with $k$ and $k+1$ tolerance algorithm. When the network is equipped with $k$-tolerance algorithm, the collisions may happen when more than $k+1$ end devices start the transmission in the same transmitting slot (as shown in slot-5). When the network is upgraded with $k+1$-tolerance algorithm, it allows maximum $k+2$ concurrent transmissions in the same slot (as shown in slot-1, slot-3, and slot-5 in Figure 6).

We estimate the overall expected SNR for both the scenarios, i.e., when the network is equipped with $k$ and $k+1$ tolerance algorithm. $\Gamma_{i}$ indicates the SNR of slot $i \in\{$ slot -1 , slot -2 , slot -3 , slot -4 , slot -5$\}$. Considering the taken example as shown in Figure 6, for $k$-tolerance algorithm scenario, the average SNR at which transmitted packet can be successful, defined as

$\Gamma_{k}^{\text {average }}=\frac{\sum_{i=1}^{5} 1_{(i, k)} \Gamma_{i}}{\sum_{i=1}^{5} 1_{(i, k)}}$,

where, $1_{(i, k)}=1$ if transmission is successful in slot $i$ otherwise 0 . Note that

$\sum_{i} 1_{(i, k)} \leq \sum_{i} 1_{(i, k+1)}$

The target packets are successfully transmitted in slots 1 and 3 with $k$-tolerance algorithm and in slots 1,3 and 5 with $k+1$-tolerance algorithm. In the considered example, the average SNR for a packet to be successful in the network when equipped with $k-$ and $k+1-$ tolerance algorithm are

$\Gamma_{k}^{\text {average }}=\frac{\Gamma_{1}+\Gamma_{3}}{2}$,

$\Gamma_{k+1}^{\text {average }}=\frac{\Gamma_{1}+\Gamma_{3}+\Gamma_{5}}{3}$,

$\Gamma_{k}^{\text {average }}>\Gamma_{k+1}^{\text {average }}$

In the considered scenario of Figure $6, \Gamma_{1}, \Gamma_{3}>\Gamma_{5}$. It is observed that as we increase $k$ tolerance in the network, we require lower SNR for a packet to be successful in a transmitting slot.

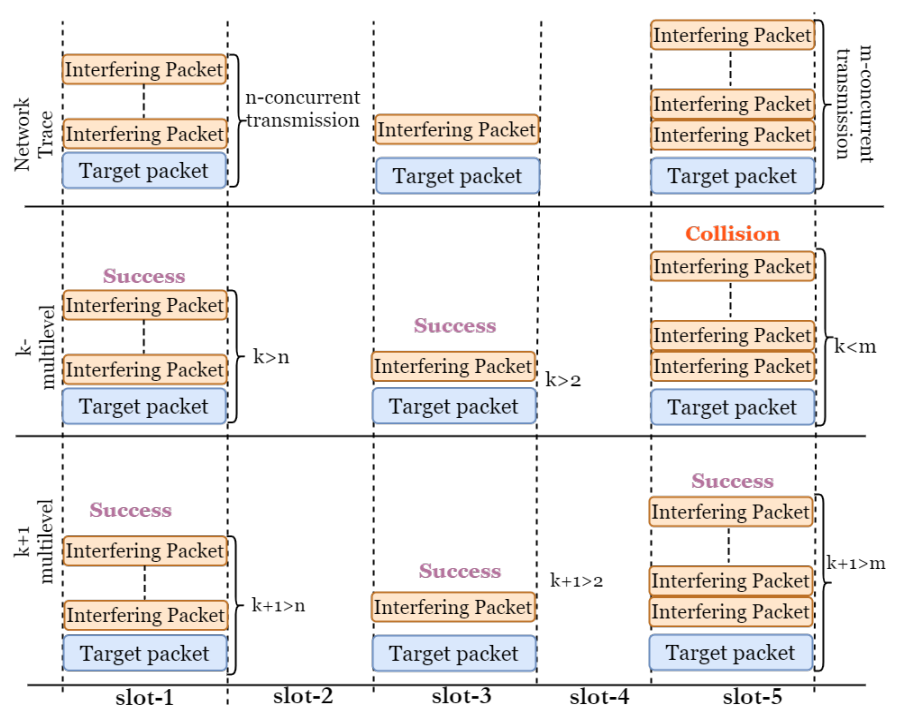

Fig. 6 Network behaviour with $k$ and $k+1$ tolerance algorithm.

Theorem 1 The rate of change of $k$ with respect to pathloss exponent $\eta$ to achieve target performance $\left(T_{p}=\right.$ $f(\eta, k))$ level is positive.

Proof The target system performance $T_{p}$ is decreases with increase in pathloss factor $\eta$ in the network with $k=0$ (i.e., no $k$-tolerance algorithm

$\frac{\partial T_{p}}{\partial \eta}<\left.0\right|_{k=0}$

By implementing the $k$-tolerance algorithm in the network, we can achieve the same target performance even with an increase in $\eta$. The $k$-tolerance algorithm allows the gateway to demodulate the transmitted packets at lower SNR.

$\Gamma_{z}^{*}=P_{0}+G_{A N T}-K_{\text {intercept }}-10 \eta \log \left(d_{z}\right)-P_{\text {noise }}$,

where $\Gamma_{z}^{*}$ is the required average SNR to be a transmitted packet successful when network equipped with $k$ 
tolerance algorithm. Considering fixed LoRaWAN cell radius $d_{z}$ (i.e., distance of an end device lies at the edge of zone $S F=12$ ) and differentiating the above equation with respect to $k$.

$\frac{d \Gamma_{z}^{*}}{d k}=-10 \log \left(d_{z}\right) \frac{d \eta}{d k}$

This $\Gamma_{z}^{*}$ has negative rate of change wrt. $k$ i.e. more value of $k$ motivates more concurrent transmission on the same channel that leads to less $S N R$ values at the gateway. Hence,

$-\frac{d \Gamma_{z}^{*}}{d k}=-10 \log \left(d_{z}\right) \frac{d \eta}{d k}$

$\frac{d \eta}{d k}=\frac{1}{10 \log \left(d_{z}\right)} \frac{d \Gamma_{z}^{*}}{d k}$

$\frac{d \eta}{d k}>0$

From the above equation, we can conclude that network with $\mathrm{k}$ tolerance can adjust to bear more pathloss to achieve the required network performance.

$\frac{d k}{d \eta}=\frac{d k}{d \rho} \frac{d \rho}{d \eta}$

Urbanization increases the node density (deployment of more IoT applications) that leads to more pathloss in the network, i.e. $\frac{d \rho}{d \eta}$ is positive. and from Figure $5, \frac{d k}{d \rho}$ is positive. Hence, $\frac{d k}{d \eta}$ is also positive. The change in target system performance over time can be countered just by installing the K-tolerance algorithm over the gateway. With the algorithm installed there is no need to change the system dimensions. The adaptation of $k$-tolerance algorithm enable the network to tolerate more pathloss and to achieve target system performance.

\section{Performance Evaluation}

To evaluate structural results of our proposed mathematical model, we use an open source simulator titled A Modular Hybrid Simulator for LoRaWAN developed by us in our previous work [25]. he simulator consists of multiple modules for different communication functionality. We adopt this simulator [31] to validate our structural results by modifying some of the simulator modules. In the considered system model, all the end devices are uniformly distributed with a centrally deployed gateway in a LoRaWAN cell of a radius $6 \mathrm{~km}$. as shown in Figure 2. The simulation results in Figure 7 show the overall success probability when the network follows our proposed network dimensions with and without $k$-tolerance algorithm and EAB. The simulation of the proposed scheme outperforms the EAB

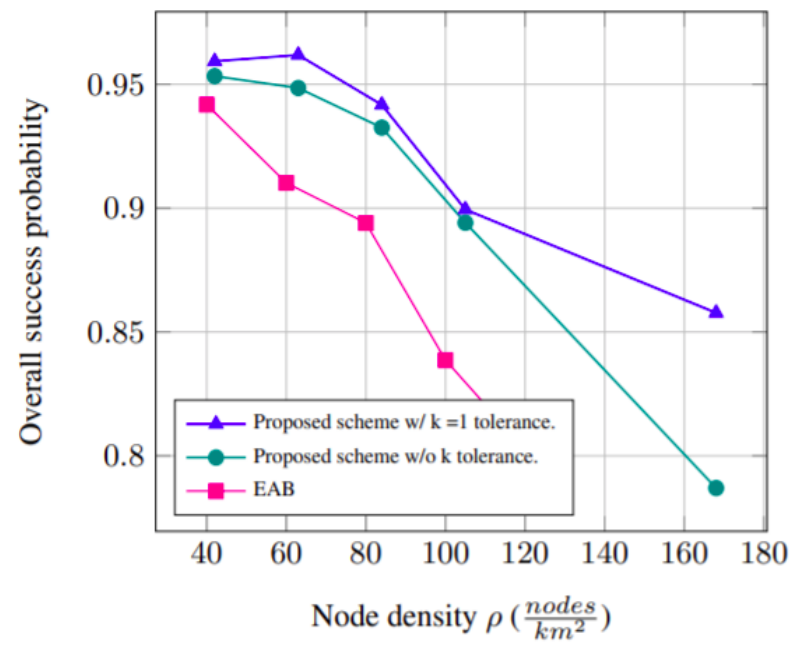

Fig. 7 Overall success probability wrt. number of nodes.

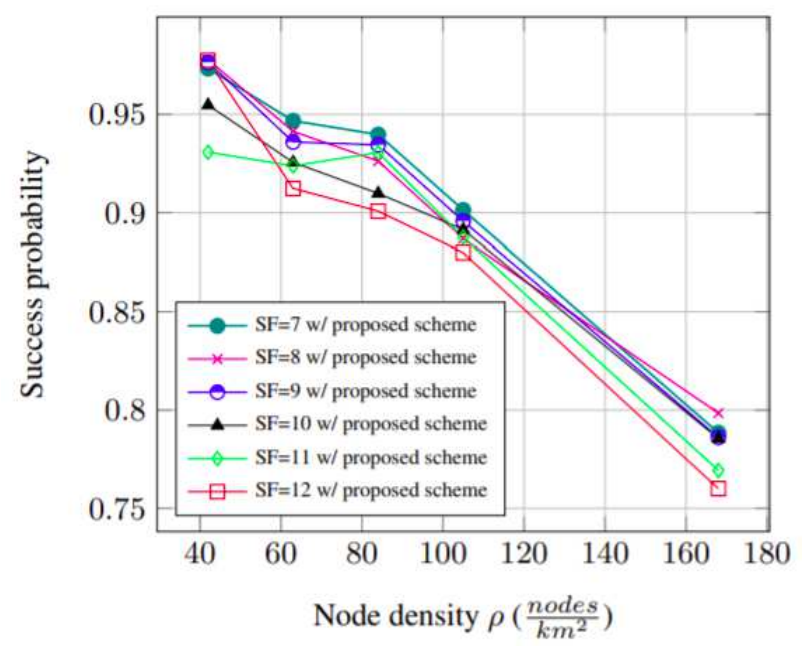

Fig. 8 Variation of Success Probability of each $S F$ zones wrt. Number of Nodes.

scheme. We observe that by adapting the $k$-tolerance algorithm in our proposed scheme, we can further enhance the overall system performance in terms of success probability. The simulation result of the proposed network dimensions in Figure 8 shows the individual end device's success probability located in different network zones. The each device's success probability is almost the same and independent of the SF usage that endorses our objective.

\section{Conclusion}

In this paper, we have proposed a mathematical framework that ensures all the end devices in each SF zone $z$ have almost same success or collision probability. The main goal of proposing this scheme is to ensure that all the end devices in the network can communicate unbi- 
asedly with the centrally deployed gateway, regardless of their location and SF usage unlike the EAB network. In the proposed mathematical framework, we estimate different SF zone boundaries to meet our objective. To validated our proposed scheme, we use an open source simulator developed by us in our previous work. The simulation of proposed scheme outperforms the LoRaWAN traditional EAB scheme. We have also presented an approach to deal with the change in network characteristics that occurs when the LoRa network region evolves from rural to urban. Urbanization causes high node density and channel load that eventually degrade the network performance. In our approach, network adopts $k$-tolerance algorithm to regain the desired system performance level.

\section{References}

1. [rp002-1.0.1 lorawan^a $\mathrm{r}$ regional parameters], available at:https://lora-alliance.org/resource-hub/rp2-101lorawanrregional- parameters- 0 .

2. LoRaWAN specifications v1.1, available at https://loraalliance.org/resource-hub/lorawanr-specification-v11 (2017)

3. available: https://loradevelopers. semtech.com/library/tech-papers-and-guides/loraandlorawan/ (2019)

4. LPWAN-Market, available at https://loradevelopers.semtech.com/library/tech-papers-andguides/lora-and-lorawan/ (2019)

5. LPWAN-Market, available at https://iotanalytics.com/5-things-to-know-about-the-lpwanmarket-in-2020 (2020)

6. Adelantado, F., Vilajosana, X., Tuset-Peiro, P., Martinez, B., Melia-Segui, J., Watteyne, T.: Understanding the limits of lorawan. IEEE Communications Magazine 55(9), 34-40 (2017). DOI 10.1109/MCOM.2017.1600613

7. Adhikary, A., Lin, X., Wang, Y..E.: Performance evaluation of nb-iot coverage. In: 2016 IEEE 84th Vehicular Technology Conference (VTC-Fall), pp. 1-5 (2016)

8. Afisiadis, O., Cotting, M., Burg, A., BalatsoukasStimming, A.: Lora symbol error rate under non-aligned interference. In: 2019 53rd Asilomar Conference on Signals, Systems, and Computers, pp. 1957-1961 (2019). DOI 10.1109/IEEECONF44664.2019.9048665

9. Amichi, L., Kaneko, M., Rachkidy, N., Guitton, A.: Spreading factor allocation strategy for lora networks under imperfect orthogonality. pp. 1-7 (2019). DOI 10.1109/ICC.2019.8761235

10. Basford, P., Bulot, F., Apetroaie-Cristea, M., Cox, S., Ossont, S.: Lorawan for smart city iot deployments: A long term evaluation. Sensors 20, 648 (2020). DOI $10.3390 / \mathrm{s} 20030648$

11. Cai, Q., Lin, J.: Improving the Scalability of LoRa Networks Through Dynamical Parameter Set Selection, vol. 1101, pp. 3-18 (2019)

12. Caillouet, C., Heusse, M., Rousseau, F.: Optimal sf allocation in lorawan considering physical capture and imperfect orthogonality. In: 2019 IEEE Global Communications Conference (GLOBECOM), pp. 1-6 (2019)
13. Casals Ibáñez, L., Mir Masnou, B., Vidal Ferré, R. Gomez, C.: Modeling the energy performance of lorawan. Sensors 17, 2364 (2017). DOI 10.3390/s17102364

14. Croce, D., Gucciardo, M., Mangione, S., Santaromita, G., Tinnirello, I.: Impact of lora imperfect orthogonality: Analysis of link-level performance. IEEE Communications Letters 22(4), 796-799 (2018). DOI 10.1109/LCOMM.2018.2797057

15. Cuomo, F., Campo, M., Caponi, A., Bianchi, G., Rossini, G., Pisani, P.: Explora: Extending the performance of lora by suitable spreading factor allocations. In: 2017 IEEE 13th International Conference on Wireless and Mobile Computing, Networking and Communications (WiMob), pp. 1-8 (2017)

16. El-Aasser, M., Elshabrawy, T., Ashour, M.: Joint spreading factor and coding rate assignment in lorawan networks. In: 2018 IEEE Global Conference on Internet of Things (GCIoT), pp. 1-7 (2018). DOI 10.1109/GCIoT.2018.8620147

17. Eletreby, R., Zhang, D., Kumar, S., Yagan, O.: Empowering low-power wide area networks in urban settings. In: SIGCOMM, pp. 309-321 (2017). DOI 10.1145/3098822.3098845

18. Elshabrawy, T., Robert, J.: Capacity planning of lora networks with joint noise-limited and interference-limited coverage considerations. IEEE Sensors Journal 19(11), 4340-4348 (2019). DOI 10.1109/JSEN.2019.2897156

19. Heusse, M., Attia, T., Caillouet, C., Rousseau, F., Duda, A.: Capacity of a lorawan cell. MSWiM '20, p. 131-140. Association for Computing Machinery, New York, NY, USA (2020). DOI 10.1145/3416010.3423228. URL https://doi.org/10.1145/3416010.3423228

20. Kherani, A.A., Maurya, K.M.P.: Improved packet detection in lora-like chirp spread spectrum systems. In: 2019 IEEE International Conference on Advanced Networks and Telecommunications Systems (ANTS), pp. 1-4 (2019). DOI 10.1109/ANTS47819.2019.9118076

21. Khutsoane, O., Isong, B., Abu-Mahfouz, A.M.: Iot devices and applications based on lora/lorawan. In: IECON 2017 - 43rd Annual Conference of the IEEE Industrial Electronics Society, pp. 6107-6112 (2017). DOI 10.1109/IECON.2017.8217061

22. Kufakunesu, R., Hancke, G., Abu-Mahfouz, A.: A survey on adaptive data rate optimization in lorawan: Recent solutions and major challenges. Sensors (Basel, Switzerland) 20 (2020). DOI 10.3390/s20185044

23. Lavric, A., Petrariu, A., Valentin, P.: Sigfox communication protocol: The new era of iot? pp. 1-4 (2019). DOI 10.1109/ISSI47111.2019.9043727

24. Mahmood, A., Sisinni, E., Guntupalli, L., Rondón, R., Hassan, S.A., Gidlund, M.: Scalability analysis of a lora network under imperfect orthogonality. IEEE Transactions on Industrial Informatics 15(3), 1425-1436 (2019). DOI 10.1109/TII.2018.2864681

25. Maurya, P., Kherani, A.A.: A modular hybrid simulator for lorawan. In: 2020 IEEE International Conference on Advanced Networks and Telecommunications Systems (ANTS), pp. 1-4 (2020)

26. Mikhaylov, K., Juha Petaejaejaervi, ., Haenninen, T. Analysis of capacity and scalability of the lora low power wide area network technology. In: European Wireless 2016; 22th European Wireless Conference, pp. 1-6 (2016)

27. Petajajarvi, J., Mikhaylov, K., Roivainen, A., Hanninen, T., Pettissalo, M.: On the coverage of lpwans: range evaluation and channel attenuation model for lora technology. In: 2015 14th International Conference on ITS 
Telecommunications (ITST), pp. 55-59 (2015). DOI 10.1109/ITST.2015.7377400

28. Rachkidy, N.E., Guitton, A., Kaneko, M.: Decoding superposed lora signals. In: 2018 IEEE 43rd Conference on Local Computer Networks (LCN), pp. 184-190 (2018). DOI 10.1109/LCN.2018.8638253

29. Singh, R., Pappinisseri, P., Berkvens, R., Weyn, M.: Energy consumption analysis of lpwan technologies and lifetime estimation for iot application. Sensors 20, 4794 (2020). DOI 10.3390/s20174794

30. Ta, D., Khawam, K., Lahoud, S., Adjih, C., Martin, S.: Lora-mab: Toward an intelligent resource allocation approach for lorawan. In: 2019 IEEE Global Communications Conference (GLOBECOM), pp. 1-6 (2019)

31. Zhu, G., Liao, C.H., Sakdejayont, T., Lai, I.W., Narusue, Y., Morikawa, H.: Improving the capacity of a mesh lora network by spreading-factor-based network clustering. IEEE Access PP, 1-1 (2019). DOI 10.1109/ACCESS.2019.2898239 